\title{
Does the association of prostate cancer with night-shift work differ according to rotating vs. fixed schedule? A systematic review and meta-analysis
}

\author{
Jennifer Mancio ${ }^{1,2} \cdot$ Cátia Leal $\mathbb{C}^{3} \cdot$ Marta Ferreira $^{3} \cdot$ Pedro Norton $^{3} \cdot$ Nuno Lunet $^{3,4}$ \\ Received: 5 November 2017 / Revised: 16 January 2018 / Accepted: 23 January 2018 / Published online: 27 April 2018 \\ (c) Macmillan Publishers Limited, part of Springer Nature 2018
}

\begin{abstract}
Background Recent studies suggested that the relation between night-shift work and prostate cancer may differ between rotating and fixed schedules.

Objectives We aimed to quantify the independent association between night-shift work and prostate cancer, for rotating and fixed schedules.

Methods We searched MEDLINE for studies assessing the association of night-shift work, by rotating or fixed schedules, with prostate cancer. We computed summary relative risk (RR) estimates with $95 \%$ confidence intervals (95\% CI) using the inverse variance method and quantified heterogeneity using the $I^{2}$ statistic. Meta-regression analysis was used to compare the summary RR estimates for rotating and fixed schedules, while reducing heterogeneity.

Results A total of nine studies assessed the effect of rotating and, in addition, four of them provided the effect of fixed nightshift work, in relation to daytime workers. Rotating night-shift work was associated with a significantly increased risk of prostate cancer ( $\mathrm{RR}=1.06,95 \% \mathrm{CI}$ of 1.01 to $1.12 ; I^{2}=50 \%$ ), but not fixed night-shift work (RR of $1.01,95 \% \mathrm{CI}$ of 0.81 to $1.26 ; I^{2}=33 \%$ ). In meta-regression model including study design, type of population, and control of confounding, the summary RR was $20 \%$ higher for rotating vs. fixed schedule, with heterogeneity fully explained by these variables.

Conclusions This is the first meta-analysis suggesting that an increased risk of prostate cancer may be restricted to workers with rotating night shifts. However, the association was weak and additional studies are needed to further clarify this relation before it can be translated into measures for risk reduction in occupational settings.
\end{abstract}

These authors contributed equally: Jennifer Mancio, Cátia Leal.

Electronic supplementary material The online version of this article (https://doi.org/10.1038/s41391-018-0040-2) contains supplementary material, which is available to authorized users.

$\triangle$ Jennifer Mancio up200104593@med.up.pt

1 Department of Surgery and Physiology, Cardiovascular Research (UnIC), Faculty of Medicine, University of Porto, Porto, Portugal

2 Department of Cardiology, Centro Hospitalar de Vila Nova de Gaia, Vila Nova de Gaia, Portugal

3 EPIUnit - Institut of Public Health, University of Porto, Porto, Portugal

4 Department of Public Health and Forensic Sciences, and Medical Education, Faculty of Medicine, University of Porto, Porto, Portugal

\section{Introduction}

In 2007, the International Agency for Research on Cancer (IARC) of the World Health Organization designated shift work involving circadian disruption as "probably carcinogenic to humans" [1]. This classification was based on evidence provided by experimental animal models and human epidemiologic studies describing an increased risk of breast cancer among long-term female night-shift workers when compared with women who do not work during the night $[2,3]$.

The association between night-shift work and cancer can possibly result from physiological changes induced by absolute sleep reduction and circadian rhythm disruption due to altered exposition to light during night, which has a worse impact in temporal alignment of genetic and metabolic processes [4]. Furthermore, pineal secretion of melatonin, a natural inhibitor of tumor growth both in vitro and in vivo, is suppressed by the exposure to artificial light in night-shift workers $[5,6]$. 
The most recent meta-analyses [7-9] examining the association between night-shift work and prostate cancer yielded an increased risk of prostate cancer among nightshift workers, with summary relative risk (RR) estimates ranging between 1.08 and 1.24. These apparently conflicting findings could result from heterogeneity between studies, which may be explained not only by differences in the study design and characteristics of the participants, but also by different night-shift work definitions and schedules, such as fixed vs. rotating.

In 2006, Kubo et al. [10] suggested, for the first time, that night-shift work related-prostate cancer risk may differ between the rotating and fixed schemes with an increased risk of prostate cancer being reported only in the rotating night-shift workers. Very recently, in a pooled analysis including studies with very different night-shift work definitions, Yong et al. supported these previous observations of higher risk of prostate cancer with rotating night-shift work. However, the authors also demonstrated an increased night-shift work related risk of prostate cancer in Asian studies, which mainly described the association with rotating night-shift work. A stratified analysis by nigh-shift work pattern is, therefore, warranted to investigate whether the differential risk estimates are due to the work schedule itself or other associated contributing factors.

In the present study, we quantified, through a systematic review and meta-analysis, the independent association of rotating and fixed night-shift work with prostate cancer, compared with daytime work, and further tested the consistency of the stratified results through exploratory metaregression analyses.

\section{Methods}

\section{Search strategy}

This study was conducted according to the Preferred Reporting Items for Systematic Reviews and Meta-analysis statement [11]. A comprehensive MEDLINE search of the literature assessing the association between night-shift work and prostate cancer was performed. In order to identify and retrieve all potentially relevant articles regarding this topic, a search was performed utilizing the following expression: [("night shift" OR "night work" OR "shift work" OR "circadian rhythm" OR "circadian disruption") AND ("cancer" OR "tumor" OR "neoplasia") AND prostate]. An additional manual search was performed through reference list of the studies selected for the systematic review, and review articles. The search was restricted to articles that were published until 17th November 2016.

\section{Eligibility criteria}

Eligible criteria in this systematic review and meta-analysis were: (1) observational studies with cohort or case-control design; (2) one of the exposures being clearly defined as rotating or fixed night-shift work; (3) prostate cancer as the dependent variable, and (4) studies providing adjusted effect size estimates with their $95 \%$ confidence intervals (CIs), or data to calculate them by rotating or fixed nightshift work. If multiple publications from the same or overlapping populations were available, the most recent or comprehensive information was included in this metaanalysis.

\section{Data extraction}

Potentially relevant articles were independently evaluated by three authors (CL, MF, and JM) using a standardized form and any disagreement was subsequently resolved by all authors. For each study, the following information was collected: first author surname, publication year, country where the study was conducted, study design, source of participants, definition of exposure, method of exposure assessment, number of incident cases, effect size (odds ratios obtained from case-control studies and hazard ratios, incidence rate ratios or standardized incidence ratios were taken as equivalent and hereafter jointly referred as RR estimates) with $95 \% \mathrm{CI}$, and covariates adjusted for in the analysis. For studies that reported several multivariable-adjusted RR estimates, we selected those accounting for the largest number of potential confounders.

\section{Statistical analysis}

Taking as reference daytime work, we calculated pooled RRs and their 95\% CIs to assess the effect of night-shift work by rotating and fixed schedule on prostate cancer with using the inverse variance method. Publication bias was assessed through visual inspection of funnel plots, the Egger's test, and the trim and fill method of Duval and Tweedie $[12,13]$. Heterogeneity between studies estimates was assessed using the $I^{2}$ statistic [14]. These analyses were conducted separately for the association between fixed and rotating night-shift schedules.

We then conducted a meta-regression analyses for a formal comparison between the summary RR estimates referring to rotating or fixed night-shift work schedule, among the studies that provided data for both exposures, while including in the models variables that may contribute to explain heterogeneity. All statistical analyses were performed using STATA software (version 13.1, StataCorp LP, Texas, US). 


\section{Results}

A total of 123 studies were initially identified, but only 13 matched our search criteria. Then, four studies were excluded because they did not include prostate cancer $(n=$ 2) [15], or did not distinguish the shift work type $(n=1)$ [16], or present overlapping data $(n=1)[17,18]$. This yielded a total of nine studies for meta-analysis (Fig. 1) [10, 17, 19-26]

Four studies provided information on associations of rotating and fixed night-shift work with prostate cancer risk in comparison with daytime work, and five studies provided information on association of rotating night-shift and prostate cancer only. Accordingly, a total of 120,319 (4.8\%) participants worked on a rotating scheme and $93,455(3.7 \%)$ on a fixed night-shift schedule. Overall, 9219 cases of prostate cancer were included. The characteristics of the included studies are shown in online Supplementary Table 1.

Figure 2 shows the forest plot of pooled risk estimates for overall, rotating, and fixed night-shift work. There was a significantly increased risk of prostate cancer with rotating (RR of $1.06,95 \%$ CI of 1.01 to $1.12 ; I^{2}=50.2 \%$ ), but not with fixed night-shift work (RR of $1.01,95 \% \mathrm{CI}$ of 0.81 to $1.26 ; I^{2}=33.3 \%$ )

Subgroup analyses by study design, study country, study population, and control of confounding by smoking or body mass index (BMI) are presented in Table 1. For the rotating schedule, stronger associations and no heterogeneity were observed in case-control studies and among Asian populations. No meaningful differences in the summary RR estimates were observed according to the control of confounding by BMI or smoking, though heterogeneity was lower when the adjusted estimates were considered. Only one study was conducted among healthcare workers, with a RR higher than the summary RR from studies conducted in industrial workers or in the general population. The results regarding the fixed schedule are based on a smaller number of studies; nevertheless, the summary RR was $<1$ for cohort studies and those presenting RR estimates adjusted for smoking or BMI. After restricting our analysis to the four abovementioned studies that reported the association between both rotating and fixed night-shift and prostate cancer, all cohort studies in which adequate control for potential confounding by smoking was performed, we found a stronger positive association, though borderline significant, between rotating night-shift work and prostate cancer (RR of $1.10,95 \%$ CI of 0.99 to $1.21 ; I^{2}=42.7 \%$ ). When comparing the summary RR for rotating and fixed schedule through meta-regression, the model including study design, type of population, and control of confounding yielded a summary RR 20\% higher for rotating schedule, compared to the fixed, with heterogeneity being fully explained by these variables $\left(I^{2}=0 \%\right)$ (Fig. 3).

The visual inspection of the funnel plots suggests no publication bias for fixed night-shift work (Egger's test, $p$ $=0.87$, but the association between rotating night-shift work and prostate cancer may be overestimated (Egger's test, $p=0.04$ ) (Fig. 4). Corresponding to the Duval and Tweedie's trim and fill input method, the expected summary RR for rotating night-shift work would be 1.05 , 95\% CI: 0.99 to 1.06 ).
Fig. 1 Systematic review flowchart

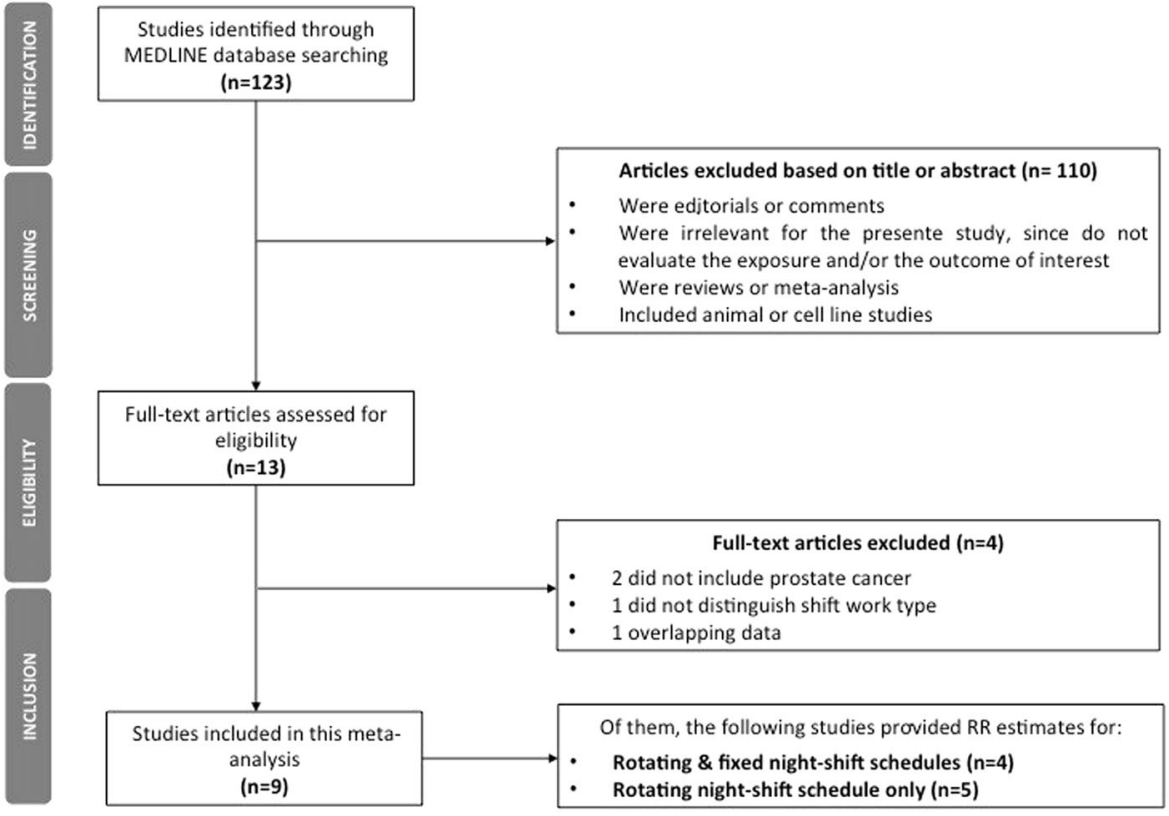


Fig. 2 Forest plot of studies $(n=9)$ describing the association of prostate cancer with rotating or fixed night-shift work. RR* describes the association between night-shift work and prostate cancer
Study

ID

$\mathrm{RR}^{*}(95 \% \mathrm{Cl})$

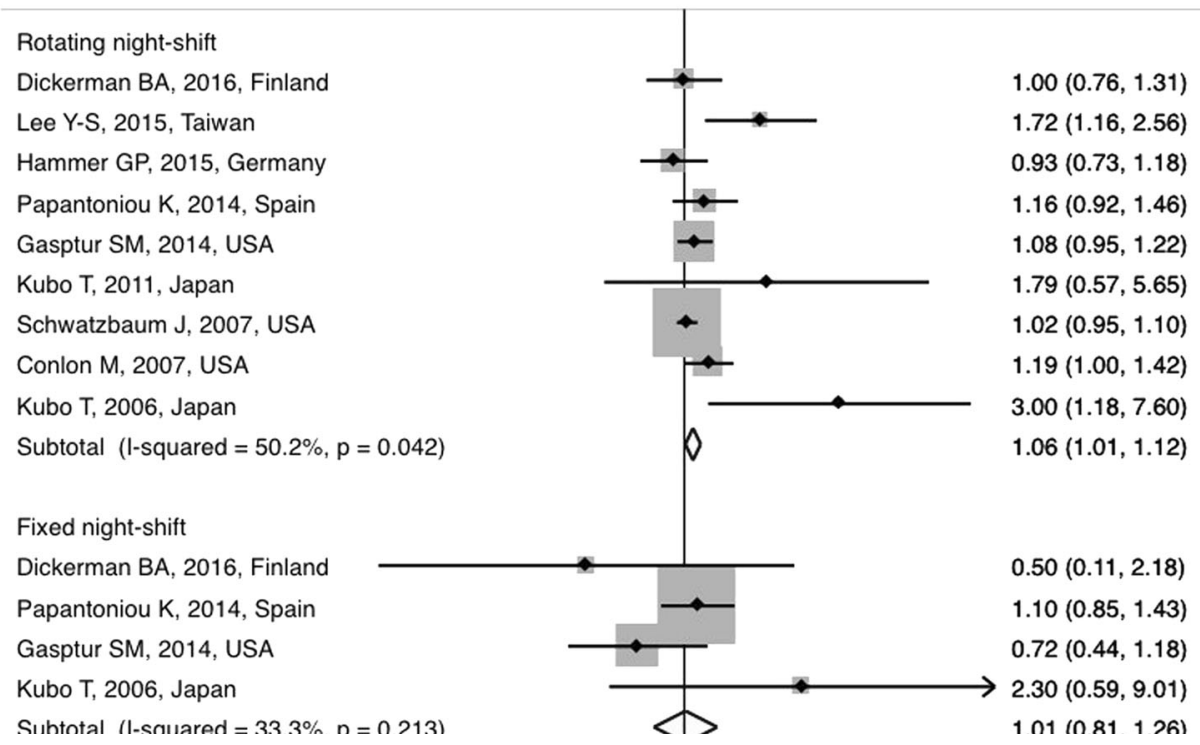

Table 1 Subgroup analyses according to studies' design, country of origin, population, and variables included in multiple regression among rotating and fixed night-shift workers

\begin{tabular}{|c|c|c|c|c|c|c|}
\hline & \multicolumn{3}{|c|}{ Rotating night-shift work } & \multicolumn{3}{|c|}{ Fixed night-shift work } \\
\hline & No. of studies & RR $(95 \% \mathrm{CI})$ & $I^{2}(\%)$ & No. of studies & RR $(95 \% \mathrm{CI})$ & $I^{2}(\%)$ \\
\hline Overall design & 9 & 1.06 (1.01 to 1.12$)$ & 50.2 & 4 & $1.01(0.81$ to 1.26$)$ & 33.3 \\
\hline Cohort & 7 & $1.04(0.98$ to 1.11$)$ & 55.8 & 3 & 0.79 (0.61 to 1.23$)$ & 30.1 \\
\hline Case-control & 2 & 1.18 (1.02 to 1.36$)$ & 0.0 & 1 & $1.10(0.84$ to 1.43$)$ & - \\
\hline \multicolumn{7}{|l|}{ Country } \\
\hline European & 2 & $1.03(0.89$ to 1.19$)$ & 0.0 & 2 & 1.07 (0.83 to 1.38$)$ & 6.4 \\
\hline North American & 4 & 1.05 (0.99 to 1.12$)$ & 27.3 & 1 & $0.72(0.44$ to 1.18$)$ & - \\
\hline Asian & 3 & 1.87 (1.32 to 2.64$)$ & 0.0 & 1 & $2.30(0.59$ to 9.01$)$ & - \\
\hline \multicolumn{7}{|l|}{ Population } \\
\hline General & 5 & 1.06 (1.00 to 1.12$)$ & 0.0 & 3 & $0.60(0.79$ to 1.24$)$ & 34.5 \\
\hline Industrial & 3 & $1.02(0.82$ to 1.29$)$ & 70.0 & 1 & $2.30(0.59$ to 9.01$)$ & - \\
\hline Healthcare & 1 & $1.72(1.16$ to 2.56$)$ & - & 0 & & \\
\hline \multicolumn{7}{|l|}{ BMI-adjusted } \\
\hline No & 5 & $1.06(0.99$ to 1.12$)$ & 60.7 & 1 & $1.10(0.84$ to 1.43$)$ & - \\
\hline Yes & 4 & 1.08 (0.97 to 1.22$)$ & 42.0 & 3 & $0.79(0.51$ to 1.23$)$ & 30.1 \\
\hline \multicolumn{7}{|l|}{ Smoking-adjusted } \\
\hline No & 3 & $1.06(0.99$ to 1.13$)$ & 75.4 & 1 & $1.10(0.84$ to 1.43$)$ & - \\
\hline Yes & 6 & 1.07 (0.98 to 1.18$)$ & 33.7 & 3 & $0.79(0.51$ to 1.23$)$ & 33.0 \\
\hline
\end{tabular}

$B M I$ body mass index, $C I$ confidence interval, $R R$ relative risk

\section{Discussion}

\section{Main finding}

In this systematic review and meta-analysis, we provide a comprehensive assessment of the factors affecting the association between night-shift work and prostate cancer, specifically the night-shift work pattern. Although some studies have suggested that rotating night-shift work, but not fixed night-shift, is associated with increased risk of prostate cancer, other studies have also attributed significant differences on the effect size to ethnicity and study design 


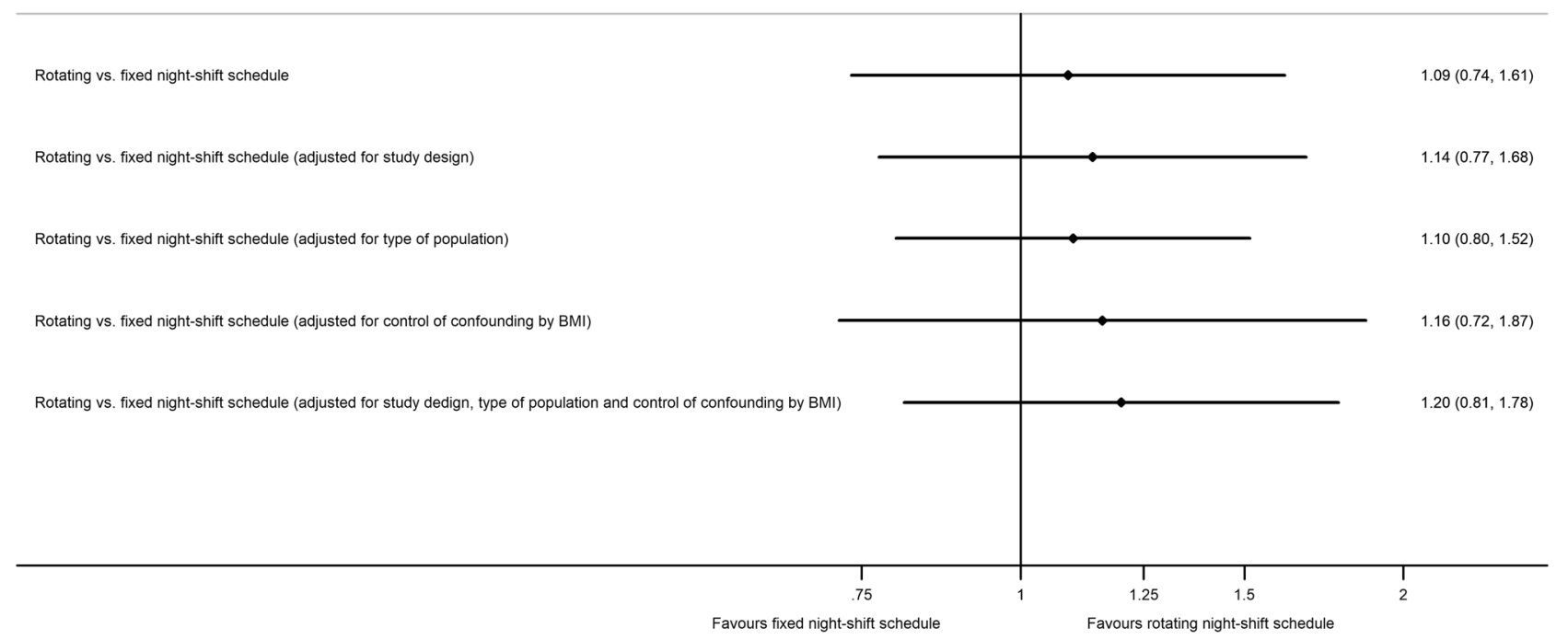

Fig. 3 Formal comparisons between the summary relative risk estimates referring to rotating or fixed night-shift work schedule, from metaregression models

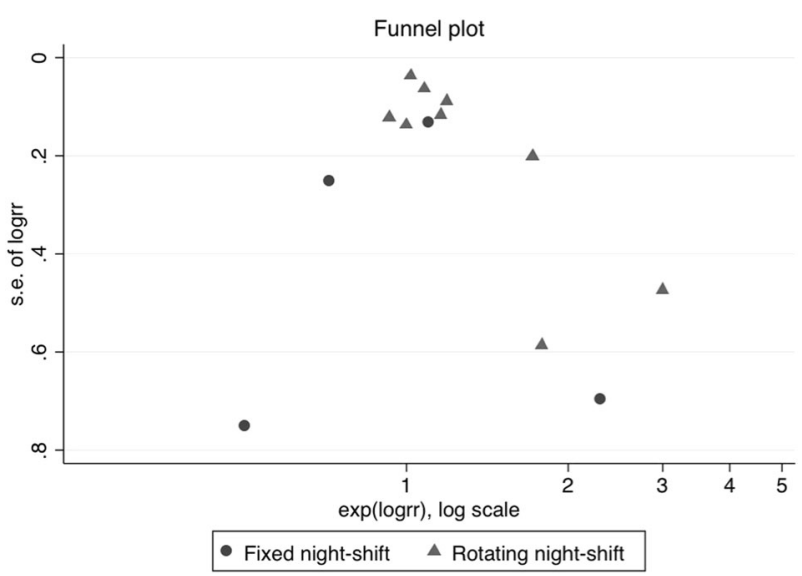

Fig. 4 Funnel plot of studies assessing the association of prostate cancer with rotating or fixed night-shift work. RR describes the association between night-shift work and prostate cancer

differences. In the present work, we included nine observational studies, in which the exposure was clearly defined as rotating and/or fixed night-shift work, and the association of with prostate cancer reported according the exposure type. We found a significant, though modest, increased risk of prostate cancer in rotating night-shift workers compared with daytime workers, while no association was found in the fixed night-shift group. This corresponded to RR estimates approximately 10 to $20 \%$ higher for rotating night shifts In addition to night-shift work schedule, heterogeneity could also be explained by study design features and characteristics of the populations evaluated.

\section{Potential biological mechanisms}

The mechanisms involved in the causal pathway between night-shift work and prostate cancer are incompletely understood, however, several biological plausible hypotheses have been formulated. Irrespective of night-shift work pattern, previous studies have shown that working at night suppresses the night-time production of melatonin [27, 28], disrupts the function of "clock" genes [27, 29], and reduces the production of vitamin $\mathrm{D}[10,30]$.

Melatonin is secreted at night by the pineal gland, and it plays an important role in the regulation of the biological circadian rhythms. Several in vitro and in vivo studies reported that melatonin has potentially beneficial oncostatic, antioxidant, antiadduct, antiestrogenic, and immune modulation activities, which are responsible for preventing cancer development, particularly in breast and prostate cancer [28, 31]. Suppression of melatonin could result in increased mutagenesis and oxidative damage, reduced DNA repair, and enhanced immune suppression with a shift in the regulation of inflammatory cytokines to those more likely to promote cancer [31]. Additionally, as melatonin suppression is believed to increase the level of sex hormones, decreased levels of melatonin may induce the continuous production of testosterone, which in turn may influence the risk of prostate cancer as the growth and differentiation of the prostate is under androgen control [10].

Apart from melatonin, other mechanisms may include the alteration of the function of "clock" genes and the desynchronization of master clocks located in the 
suprachiasmatic nucleus ( $\mathrm{SCN}$ ) and peripheral clocks in tissues. Desynchronization of the SCN and peripheral clocks by a circadian phase shift may lead to abolishment of peripheral-clock-controlled ATM (ataxia teleangiectasia mutated) gene activation, with impaired DNA repair and activation of c-Myc oncogenic potential. Consequently, tumor suppression is a clock-controlled process with interplay between central and peripheral oscillators, and disruption of the circadian time organization favors tumor promotion and tumor development. In addition, short sleep duration can also affect the circadian cycle resulting in the dysregulation of a number of genes involved in tumor suppression. "Clock" genes are known to be directly involved in the regulation of prostate tumorigenesis. In normal individuals, the "clock" genes, especially Perl/ per 2 and Bmall, are expressed in a circadian rhythmic manner. This rhythmicity is lost in patients with prostate cancer, and melatonin can regulate Bmall levels, restoring circadian function $[32,33]$.

Finally, the decreased exposure to daylight imposed by night-shift work reduced the production of vitamin $\mathrm{D}$, and the biological active form of vitamin D (1,25-dihydroxyvitamin D3) was shown to inhibit prostate cancer cells proliferation [30]. Beyond its primary role of bone metabolism regulation and calcium-phosphorus homeostasis, numerous in vitro and in vivo studies have evidenced several "noncalcemic" or "extraskeletal" effects of vitamin D. Reduced levels of vitamin D influence the onset and progression of several diseases such as autoimmune diseases, respiratory infections, diabetes mellitus type 1 and 2, hypertension and cardiovascular diseases, neuromuscular disorders, and cancer [34]. Calcitriol exerts significant antitumoral activity in vitro and in vivo in murine squamous cell carcinoma, rat metastatic prostatic adenocarcinoma Dunning (MLL) model systems, human prostatic adenocarcinoma (PC-3 and LNCaP), human breast, colon, and pancreatic cancer, as well as in leukemia, myeloma, and lymphoma lines [35]. The value of vitamin D or vitamin D analogs in the prevention and treatment of prostate cancer have generated great interest and its efficacy tested in recent clinical trials with promising results $[36,37]$.

\section{Causes of heterogeneity: night-shift schedule, study population, confounding, and bias}

Regarding the influence of different night-shift schedules on circadian rhythms disruption, Borugian et al. [38] compared the melatonin levels between rotating-shift workers and fixed shift workers. These levels were lower and less variable in rotating night-shift workers, with low levels during rest/sleep periods and high levels during waking/work, as compared with those who works permanently at night. These authors showed that high melatonin levels during waking and working hours are the main cause of fatigue and lack of alertness in shift workers, while the decreased levels of melatonin during sleep are possibly related with known increased cancer risk. These results suggest that fast-rotating shifts may not allow sufficient time for intrinsic circadian clock to adapt, whereas permanent night workers are allowed to adapt almost completely to their sleep/wake cycle, provided they maintain it even on their days-off. The higher and statistically significant risk of cancer that we observed in rotating night-shift workers compared with fixed night-shift workers, can possibly result from a more severe disruption of the circadian rhythm, reflected by lower melatonin levels when compared with the permanent work at night, and consequently a greater risk of tumorigenesis than the fixed night-shift workers. In addition, among those working in a rotating nigh-shift we showed a stronger association in Asian, and industrial workers. These observations might reflect Asian specificgenetic susceptibilities leading to an inappropriate adaptation to night-shift; since the only study including healthcare workers were Asian we cannot exclude collinearity between study location and population; in general population studies, however, the association with rotating night-shift work and prostate cancer were also positive, though less significant. The hypothesis of confounding by smoking and obesity was taken into account in statistical analysis of the majority of studies, and thus they did not affect effect size heterogeneity between studies.

\section{Study limitations and strengths}

One of the distinctive features of the present systematic review in relation with previous reports addressing the effect of night-shift work on prostate cancer is the specific focus on the effects of fixed or rotating schedules. However, it is subject to the potential limitations of this type of analysis. We did not have access to individual patient data from all studies reviewed and we based on published information only. Despite the high number of participants, most of them were recruited from general population and there are few studies specifically addressing the association between rotating or fixed night-shift work and prostate cancer. Additionally, the definition of the exposure differs largely between studies. Nevertheless, and importantly, the level of heterogeneity in this review was lower than those reported in the previous studies, which suggests that a stratified analysis is needed when consider the effect of night-shift work on the risk of cancer. The total number of participants exposed (i.e., nightshift workers) was $<10 \%$ of the non-exposed (i.e., daytime workers). However, the distribution of participants was similar for rotating and fixed schedule, and consequently the statistical power for detecting association of a similar magnitude is similar for both exposures, despite the number of studies providing data for fixed schedule is smaller. The 
original studies do not provide a direct comparison between the effects of fixed and rotating schedules, and the $95 \% \mathrm{CI}$ for the summary RR estimates for fixed and rotating shifts overlap. Although our findings cannot support statistically significant differences between these distinct types of exposure to night-shift work, in the four studies that provided data for both fixed and rotating schedule the association was consistently stronger for the latter. Also, meta-regression analyses support higher RR estimates for rotating schedule when taking into account characteristics of the studies that could explain such differences. However, data on the effects of rotating night-shift work may have been less likely to be published when there was no positive association, which may have contributed for an overestimation of the summary effect, though to a small extent.

It would have been interesting to investigate if specific night-shift work pattern affect prostate cancer according to the stage or aggressiveness of the tumors, but unfortunately such data is seldom available in the original reports, and no RR estimates are provided according to these tumor characteristics.

\section{Conclusion and public policy implications}

The present systematic review and meta-analysis provides new evidence into the field of occupational health and prostate cancer research. Its findings may improve our understanding of the role of circadian rhythm disruption in prostate cancer and may help to shed light on potential risk factors for prostate cancer. It shows that, when compared with the daytime workers, in men who work at night the rotating schedule was associated with a significantly increased risk of prostate cancer, but not the fixed schedule. However, since the association was weak and residual confounding or bias may have contributed to spuriously increased risks, additional studies are needed to further clarify this association before this evidence can be translated into measures for risk reduction in occupational settings.

Funding Mancio $\mathrm{J}$ is supported by the Fundação Portuguesa para a Ciência e Tecnologia (SFRH/BD/104369/2014) and by the Sociedade Portuguesa de Cardiologia ("Bolsa de investigação João Porto").

\section{Compliance with ethical standards}

Conflict of interest The authors declare that they have no conflict of interest.

\section{References}

1. Straif K, Baan R, Grosse Y, Secretan B, El Ghissassi F, Bouvard $\mathrm{V}$, et al. Carcinogenicity of shift-work, painting, and fire-fighting. Lancet Oncol. 2007;8:1065-6.
2. Megdal SP, Kroenke CH, Laden F, Pukkala E, Schernhammer ES. Night work and breast cancer risk: a systematic review and metaanalysis. Eur J Cancer. 2005;41:2023-32.

3. Schernhammer ES, Kroenke CH, Laden F, Hankinson SE. Night work and risk of breast cancer. Epidemiol (Camb, Mass). 2006;17:108-11.

4. Filipski E, Lévi F. Circadian disruption in experimental cancer processes. Integr Cancer Ther. 2009;8:298-302.

5. Sainz RM, Mayo JC, Dx Tan, León J, Manchester L, Reiter RJ. Melatonin reduces prostate cancer cell growth leading to neuroendocrine differentiation via a receptor and PKA independent mechanism. Prostate. 2005;63:29-43.

6. Zhou W, Zhang X, Zhu C-L, He Z-Y, Liang J-P, Song Z-C. Melatonin receptor agonists as the "perioceutics" agents for periodontal disease through modulation of porphyromonas gingivalis virulence and inflammatory response. PLoS ONE. 2016;11: $\mathrm{e} 0166442$.

7. Du HB, Bin KY, Liu WH, Yang FS. Shift work, night work, and the risk of prostate cancer: A meta-analysis based on 9 cohort studies. Medicine. 2017;96:e8537.

8. Gan Y, Li L, Zhang L, et al. Association between shift work and risk of prostate cancer: a systematic review and meta-analysis of observational studies. Carcinogenesis. 2018;39:87-97.

9. Rao D, Yu H, Bai Y, Zheng X, Xie L. Does night-shift work increase the risk of prostate cancer? a systematic review and metaanalysis. Onco Targets Ther. 2015;8:2817-26.

10. Kubo T, Ozasa K, Mikami K, Wakai K, Fujino Y, Watanabe Y, et al. Prospective cohort study of the risk of prostate cancer among rotating-shift workers: findings from the Japan collaborative cohort study. Am J Epidemiol. 2006;164:549-55.

11. Moher D, Liberati A, Tetzlaff J, Altman DG. Preferred reporting items for systematic reviews and meta-analyses: the PRISMA statement. Ann Intern Med. 2009;151:264-9.

12. Borenstein M, Hedges LV, Higgins J, Rothstein HR. Introduction to Meta-analysis. Front matter: Wiley Online Library; 2009. https://doi.org/10.1002/9780470743386.

13. Begg CB, Mazumdar M. Operating characteristics of a rank correlation test for publication bias. Biometrics. 1994;50:1088-101.

14. Higgins J, Thompson SG. Quantifying heterogeneity in a metaanalysis. Stat Med. 2002;21:1539-58.

15. Hsu FM, Hou WH, Huang CY, Wang CC, Tsai CL, Tsai YC, et al. Differences in toxicity and outcome associated with circadian variations between patients undergoing daytime and evening radiotherapy for prostate adenocarcinoma. Chronobiol Int. 2016;33:210-9.

16. Parent ME, El-Zein M, Rousseau MC, Pintos J, Siemiatycki J. Night work and the risk of cancer among men. Am J Epidemiol. 2012;176:751-9.

17. Yong M, Nasterlack M, Messerer P, Oberlinner C, Lang S. A retrospective cohort study of shift work and risk of cancer-specific mortality in German male chemical workers. Int Arch Occup Environ Health. 2014;87:175-83.

18. Sigurdardottir LG, Markt SC, Rider JR, Haneuse S, Fall K, Schernhammer ES, et al. Urinary melatonin levels, sleep disruption, and risk of prostate cancer in elderly men. Eur Urol. 2015;67:191-4.

19. Dickerman BA, Markt SC, Koskenvuo M, Hublin C, Pukkala E, Mucci LA, et al. Sleep disruption, chronotype, shift work, and prostate cancer risk and mortality: a 30-year prospective cohort study of Finnish twins. Cancer Causes Control. 2016;27: 1361-70.

20. Lee Y-S, Hsu C-C, Weng S-F, Lin H-J, Wang J-J, Su S-B, et al. Cancer incidence in physicians: A Taiwan National Populationbased Cohort Study. Medicine. 2015;94:e2079.

21. Hammer GP, Emrich K, Nasterlack M, Blettner M, Yong M. Shift work and prostate cancer incidence in industrial workers: A 
Historical Cohort Study in a German chemical company. Dtsch Arztebl Int. 2015;112:463.

22. Papantoniou K, Castaño-Vinyals G, Espinosa A, Aragonés N, Pérez-Gómez B, Burgos J, et al. Night shift work, chronotype and prostate cancer risk in the MCC-Spain case-control study. Int J Cancer. 2015;137:1147-57.

23. Kubo T, Oyama I, Nakamura T, Kunimoto M, Kadowaki K, Otomo $\mathrm{H}$, et al. Industry-based retrospective cohort study of the risk of prostate cancer among rotating-shift workers. Int J Urol. 2011;18:206-11.

24. Schwartzbaum J, Ahlbom A, Feychting M. Cohort study of cancer risk among male and female shift workers. Scand J Work Environ Health. 2007;33:336-43.

25. Gapstur SM, Diver WR, Stevens VL, Carter BD, Teras LR, Jacobs EJ. Work schedule, sleep duration, insomnia, and risk of fatal prostate cancer. Am J Prev Med. 2014;46:S26-33.

26. Conlon M, Lightfoot N, Kreiger N. Rotating shift work and risk of prostate cancer. Epidemiol (Camb, Mass). 2007;18:182-3.

27. Haus E, Smolensky M. Biological clocks and shift work: circadian dysregulation and potential long-term effects. Cancer Causes Control. 2006;17:489-500.

28. Sigurdardottir LG, Valdimarsdottir UA, Fall K, Rider JR, Lockley SW, Schernhammer E, et al. Circadian disruption, sleep loss, and prostate cancer risk: a systematic review of epidemiologic studies. Cancer Epidemiol Biomark Prev. 2012;21:1002-11.

29. James FO, Cermakian N, Boivin DB. Circadian rhythms of melatonin, cortisol, and clock gene expression during simulated night shift work. Sleep. 2007;30:1427-36.

30. Itoh H, Weng Z, Saito H, Ogawa Y, Nakayama K, HasegawaOhira M, et al. Association between night-shift work and serum 25-hydroxyvitamin D levels in Japanese male indoor workers: a cross-sectional study. Ind Health. 2011;49:658-62.

31. Chiru AZ, Popescu C, Gheorghe D. Melatonin and cancer. J Med Life. 2014;7:373.

32. Haus EL, Smolensky MH. Shift work and cancer risk: potential mechanistic roles of circadian disruption, light at night, and sleep deprivation. Sleep Med Rev. 2013;17:273-84.

33. Savvidis C, Koutsilieris M. Circadian rhythm disruption in cancer biology. Mol Med. 2012;18:1249-60.

34. Ombra MN, Paliogiannis P, Doneddu V, Sini MC, Colombino M, Rozzo C, et al. Vitamin D status and risk for malignant cutaneous melanoma: recent advances. Eur J Cancer Prev: Off J Eur Cancer Prev Organ (ECP). 2017;26:532-41.

35. Holick MF, Binkley NC, Bischoff-Ferrari HA, Gordon CM, Hanley DA, Heaney RP, et al. Evaluation, treatment, and prevention of vitamin D deficiency: an Endocrine Society clinical practice guideline. J Clin Endocrinol Metab. 2011;96:1911-30.

36. Jarrard D, Konety B, Huang W, Downs T, Kolesar J, Kim KM, et al. Phase IIa, randomized placebo-controlled trial of single high dose cholecalciferol (vitamin D3) and daily Genistein (G2535) versus double placebo in men with early stage prostate cancer undergoing prostatectomy. Am J Clin Exp Urol. 2016;4:17-27.

37. Wagner D, Trudel D, Van der Kwast T, Nonn L, Giangreco AA, Li D, et al. Randomized clinical trial of vitamin D3 doses on prostatic vitamin D metabolite levels and ki67 labeling in prostate cancer patients. J Clin Endocrinol Metab. 2013;98:1498-507.

38. Borugian MJ, Gallagher RP, Friesen MC, Switzer TF, Aronson KJ. Twenty-four-hour light exposure and melatonin levels among shift workers. J Occup Environ Med. 2005;47:1268-75. 